\title{
On a class of Leibniz algebras
}

\author{
Côme J.A.BÉRÉ ${ }^{1 *}$, Aslao KOBMBAYE ${ }^{2}$, Amidou KONKOBO ${ }^{1}$ \\ ${ }^{1}$ Laboratory T.N. AGATA Department of Mathematics and Computer Science \\ University of Ouagadougou Burkina Faso, Address 03 B.P. 7021 Ouagadougou 03 Burkina Faso. \\ 2 Laboratory T.N. AGATA Department of Mathematics University of Djamena Tchad, Address B.P. 1027 Djamena Tchad. \\ *Corresponding author E-mail: come_bere@univ-ouaga.bf
}

Copyright (C2015 Côme J.A. BÉRÉ et al. This is an open access article distributed under the Creative Commons Attribution License, which permits unrestricted use, distribution, and reproduction in any medium, provided the original work is properly cited.

\begin{abstract}
We pointed out the class of Leibniz algebras such that the Killing form is non degenerate implies algebras are semisimple.
\end{abstract}

Keywords: Killing form; Leibniz algebras; Leibniz modules; Representations; Semisimplicity.

\section{Introduction}

Throughout this paper, $F$ will be an algebraically closed field of characteristic zero. All vector spaces and algebras will be finite dimensional over $F$. Note the sum of two vector subspaces $V_{1}, V_{2}$ by $V_{1} \dot{+} V_{2}$ and direct sum by $V_{1} \oplus V_{2}$. It is well-known that a Lie algebra is semisimple if and only if its Killing form is non degenerate. An equivalent criterion is found for Leibniz algebra $L$ which satisfies, for all $x, y$ in $L$, the trace of the endomorphism $\left(a d_{x} \circ a d_{y}\right)_{\mid E s s(L)}$ equals zero. Call such algebras "Killing- Leibniz-Algebra".

Section 2 is devoted to basic facts. In Section 3, the links between radical and nilradical are set. Section 4 is devoted to the nilpotency of the ideal $\{\operatorname{Rad}(L), L\}$. In Section 5, the main theorem is settled. For conclusion, we give an hierarchy of Leibniz algebras and two questions are done about Killing Leibniz Algebras.

\section{Preliminary notes}

Let us note that Leibniz algebras are defined in two classes:

- Right Leibniz algebras, with the rule

$$
[x,[y, z]]=[[x, y], z]-[[x, z], y] \text { for any } x, y, z \in L .
$$

- Left Leibniz algebras, with the rule

$$
[x,[y, z]]=[[x, y], z]+[y,[x, z]] \text { for any } x, y, z \in L .
$$

For an algebra $(A,[]$,$) with vectors multiplication [a, b]$, for all $a, b$ in $A$, define the algebra $\left(A,[,]^{o p}\right)$ as the underlying vector space $A$ where the vectors multiplication is defined by $[a, b]^{o p}=[b, a]$. We have that: 
Proposition 2.1. The algebra $(A,[]$,$) is left Leibniz algebra if and only if the algebra \left(A,[,]^{o p}\right)$ is right Leibniz algebra.

So results on Left Leibniz algebras are available on Right Leibniz algebras, (with minors variations).

Here we write "Leibniz algebras" for "Right Leibniz algebras".

It follows from the equation (1) called Leibniz identity that in any Leibniz algebra one has

$$
[y,[x, x]]=0,[z,[x, y]]+[z,[y, x]]=0, \text { for all } x, y, z \in L .
$$

Definition 2.2. (Ideal) A subspace $H$ of a Leibniz algebra $L$ is called left (respectively right) ideal if for a $\in H$ and $x \in L$ one has $[x, a] \in H$ (respectively $[a, x] \in H$ ). If $H$ is both left and right ideal, then $H$ is called (two-sided) ideal.

If $V$ is a vector space, let $\operatorname{End}_{F}(V)$ denotes the set of all endomorphisms of $V$. An action of $L$ on $\operatorname{End}_{F}(V)$ is a linear map of $L$ on $\operatorname{End}_{F}(V)$.

Definition 2.3. (Representation) Let $L$ be a Leibniz algebra and $V$ a vector space. $V$ is an L-module if there are:

- a left action, $l: L \longrightarrow \operatorname{End}_{F}(V), x \mapsto l_{x}$,

- a right action, $r: L \longrightarrow \operatorname{End}_{F}(V), x \mapsto r_{x}$, such that:

$$
\begin{aligned}
r_{[x, y]} & =r_{y} r_{x}-r_{x} r_{y}, \\
l_{[x, y]} & =r_{y} l_{x}-l_{x} r_{y}, \\
l_{[x, y]} & =r_{y} l_{x}+l_{x} l_{y} .
\end{aligned}
$$

For $x$ in $L, r_{x}(v)$ will be denoted by $v x$ and $l_{x}(v)$ will be denoted by $x v$. The triplet $(l, r, V)$ is called a representation of $L$ on $V$. Now if $L$ is a Leibniz algebra, we have the adjoint representation " $(A d, a d, L)$ " defined as follows: for all $x$ and $y$ in $L, a d_{x}: L \longrightarrow L, y \longmapsto[y, x]$ and $A d_{x}: L \longrightarrow L, y \longmapsto[x, y]$.

\section{Remark 2.4.}

For $x \in L, a d_{x}: L \longrightarrow L$ is a derivation of $L$ i.e. for all $x, y, z$ in $L, a d_{x}([y, z])=\left[a d_{x}(y), z\right]+\left[y, a d_{x}(z)\right]$. For $x \in L, A d_{x}: L \longrightarrow L$ is an anti-derivation of $L$ i.e. for all $x, y, z$ in $L, A d_{x}([y, z])=\left[\operatorname{Ad}_{x}(y), z\right]-\left[\operatorname{Ad}_{x}(z), y\right]$.

For an arbitrary algebra and for all non negative integer $n$ let us define the sequences:

(i) $D^{1}(L)=L^{[1]}=L^{2}, D^{n+1}(L)=L^{[n+1]}=\left[L^{[n]}, L^{[n]}\right]$;

(ii) $L^{1}=L, L^{n+1}=\left[L^{1}, L^{n}\right]+\left[L^{2}, L^{n-1}\right]+\cdots+\left[L^{n-1}, L^{2}\right]+\left[L^{n}, L^{1}\right]$.

Definition 2.5. ([1]) An algebra $L$ is called solvable if there exists $m \in \mathbb{N}^{*}$ such that $D^{m}(L)=L^{[m]}=\{0\}$. An algebra $L$ is called nilpotent if there exists $m \in \mathbb{N}^{*}$ such that $L^{m}=\{0\}$.

Definition 2.6. Let $A$ be a subspace of a Leibniz algebra $L$. The normalizer of $A$ is denoted by:

$$
n_{L}(A)=\{y \in L \mid[y, a] \in A \text { and }[a, y] \in A \text { for all } a \in A\} .
$$

Definition 2.7. ([4]) A Leibniz algebra $L$ is said to be semisimple if $\operatorname{Rad}(L)=\operatorname{Ess}(L)$.

Equivalently, we can say that:

Leibniz algebra $L$ simple if $\{0\} \neq[L, L] \neq \operatorname{Ess}(L)$ and every ideal of $L$ belongs to the set $\{L, E s s(L),(0)\}$.

Since $D \imath=\imath^{2}$ is an ideal whenever $\imath$ is (by Equation 1 ), if $\operatorname{rad}(L) \neq \operatorname{Ess}(L)$ then $L$ contains an ideal $\jmath$ which satisfies $\jmath^{2} \subseteq E s s(L) \subsetneq \jmath$.

So an other equivalent definition is:

Remark 2.8. $L$ is semisimple if it has no ideal $\jmath$ which satisfies $\jmath^{2} \subseteq \operatorname{Ess}(L) \subsetneq \jmath$.

Lemma 2.9. [3] Let $L$ be a Leibniz algebra and $(l, r, V)$ a representation of $L$. Let $A$ be a subspace of $L$, then $r_{A}=\left\{r_{x}\right.$, for all $\left.x \in A\right\}$ is a subspace of the vector space $\operatorname{End}_{F}(V)$. In particular, $r_{L}$ is a Lie subalgebra of $g l(V)$ and $L$ is solvable (respectively nilpotent) if and only if $r_{L}$ is solvable (respectively nilpotent).

Proof. The results are clear since for all $x, y$ in $L$ and for all $\lambda$ in $F$, we have that $r_{x+\lambda y}=r_{x}+\lambda r_{y}$ and $\left[r_{x}, r_{y}\right]=r_{[y, x]}$. 
Remark 2.10. Let $L$ be a Leibniz algebra and $(l, r, V)$ a representation of $L$. If for all $x$ in $L, r_{x}$ is nilpotent then $l_{x}$ is also nilpotent for all $x$. Since we have $l_{x}^{k}=(-1)^{k+1} l_{x}\left(r_{x}\right)^{k-1}$. Thus when $r_{x}$ is nilpotent for all $x$ in L, we can say that the representation $(l, r, V)$ of $L$ is nilpotent.

Lemma 2.11. Let $L$ be a Leibniz algebra and $(l, r, V)$ a representation of $L$. Let $A$ be a subspace of the vector space $L$ and let $x$ in the normalizer $n_{L}(A)$ of $A$. Then we have for all integer $k$ in $\mathbb{N}$ and for all a in $A$ :

i) $\delta_{k+1}=r_{a}^{k+1} r_{x}-r_{x} r_{a}^{k+1} \in r_{A}^{k+1}$.

ii) $\beta_{k+1}=r_{x}^{k+1} r_{a}-r_{a} r_{x}^{k+1} \in r_{A} r_{x}^{k} \dot{+} \cdots \dot{+} r_{A} r_{x} \dot{+} r_{A}$.

Proof. For i), since $\left[r_{a}, r_{x}\right]=r_{[x, a]}$, we have $\delta_{1}=r_{a} r_{x}-r_{x} r_{a}=r_{[x, a]}$. Thus $\delta_{1} \in r_{A}$ since $x \in n_{L}(A)$. And we have:

$$
\begin{aligned}
\delta_{2} & =r_{a}^{2} r_{x}-r_{x} r_{a}^{2}=r_{a}\left(r_{a} r_{x}\right)-r_{x} r_{a}^{2}=r_{a}\left(r_{x} r_{a}+\delta_{1}\right)-r_{x} r_{a}^{2}=\left(r_{a} r_{x}\right) r_{a}+r_{a} \delta_{1}-r_{x} r_{a}^{2} \\
& =\left(r_{x} r_{a}+\delta_{1}\right) r_{a}+r_{a} \delta_{1}-r_{x} r_{a}^{2}=\delta_{1} r_{a}+r_{a} \delta_{1} \in r_{A}^{2} .
\end{aligned}
$$

With the hypothesis of recurrence: $\delta_{k}=r_{a}^{k} r_{x}-r_{x} r_{a}^{k} \in r_{A}^{k}$, we get:

$$
\begin{aligned}
\delta_{k+1} & =r_{a}^{k+1} r_{x}-r_{x} r_{a}^{k+1}=r_{a}\left(r_{a}^{k} r_{x}\right)-r_{x} r_{a}^{k+1}=r_{a}\left(r_{x} r_{a}^{k}+\delta_{k}\right)-r_{x} r_{a}^{k+1}=\left(r_{a} r_{x}\right) r_{a}^{k}+r_{a} \delta_{k}-r_{x} r_{a}^{k+1} \\
& =\left(r_{x} r_{a}+\delta_{1}\right) r_{a}^{k}+r_{a} \delta_{k}-r_{x} r_{a}^{k+1}=\delta_{1} r_{a}^{k}+r_{a} \delta_{k} \in\left(r_{A}\right)^{k+1} .
\end{aligned}
$$

And for ii), we have $\left[r_{x}, r_{a}\right]=r_{[a, x]}$, so $\beta_{1}=-\delta_{1} \in r_{A}=r_{A} r_{x}^{0}$ since $x \in n_{L}(A)$ (where $\left.r_{x}^{0}=1_{V}\right)$. Note that we have:

$$
\begin{aligned}
\beta_{2} & =r_{x}^{2} r_{a}-r_{a} r_{x}^{2}=r_{x}\left(r_{x} r_{a}\right)-r_{a} r_{x}^{2}=r_{x}\left(r_{a} r_{x}+r_{[a, x]}\right)-r_{a} r_{x}^{2}=\left(r_{x} r_{a}\right) r_{x}+r_{x} r_{[a, x]}-r_{a} r_{x}^{2} \\
& =\left(r_{a} r_{x}+r_{[a, x]}\right) r_{x}+\left(r_{[a, x]} r_{x}+r_{[[a, x], x]}\right)-r_{a} r_{x}^{2}=2 r_{[a, x]} r_{x}+r_{[[a, x], x]} \in r_{A} r_{x} \dot{+} r_{A} .
\end{aligned}
$$

Set the hypothesis that $\beta_{k}=r_{x}^{k} r_{a}-r_{a} r_{x}^{k} \in r_{A} r_{x}^{k-1} \dot{+} \cdots \dot{+} r_{A} r_{x} \dot{+} r_{A}$, and then it will follow that:

$$
\begin{aligned}
\beta_{k+1} & =r_{x}^{k+1} r_{a}-r_{a} r_{x}^{k+1}=r_{x}^{k}\left(r_{x} r_{a}\right)-r_{a} r_{x}^{k+1}=r_{x}^{k}\left(r_{a} r_{x}+r_{[a, x]}\right)-r_{a} r_{x}^{k+1}=\left(r_{x}^{k} r_{a}\right) r_{x}+r_{x}^{k} r_{[a, x]}-r_{a} r_{x}^{k+1} \\
& =\left(r_{a} r_{x}^{k}+\beta_{k}\right) r_{x}+r_{[a, x]} r_{x}^{k}+\beta_{1}^{\prime}-r_{a} r_{x}^{k+1}\left(\text { where } \beta_{1}^{\prime}=r_{x}^{k} r_{[a, x]}-r_{[a, x]} r_{x}^{k}=r_{[[a, x], x]}^{k} \in r_{A}^{k}\right) \\
& =\beta_{k} r_{x}+r_{[a, x]} r_{x}^{k}+\beta_{1}^{\prime} \in\left(r_{A} r_{x}^{k-1} \dot{+} \cdots \dot{+} r_{A}\right) r_{x}+r_{A} r_{x}^{k}+\dot{+} r_{A} \in r_{A} r_{x}^{k} \dot{+} r_{A} r_{x}^{k-1} \dot{+} \cdots+r_{A} r_{x} \dot{+} r_{A} .
\end{aligned}
$$

Proofs are done.

Lemma 2.12. Let $L$ be a Leibniz algebra and $(l, r, V)$ a representation of $L$. Let $A$ be a subspace of the vector space $L$ and $x$ in the normalizer $n_{L}(A)$ of $A$. Then we have for all integer $k$ and $p$ in $\mathbb{N}$ :

$$
\left[r_{A}^{p} r_{x}^{k}\right] \circ r_{A} \subseteq r_{A}^{p+1} r_{x}^{k} \dot{+} \cdots \dot{+} r_{A}^{p+1} r_{x} \dot{+} r_{A}^{p+1}
$$

Proof. We shall note that:

$$
\left[r_{A}^{p} r_{x}^{k}\right] \circ r_{A}=r_{A}^{p} \circ\left[r_{x}^{k} \circ r_{A}\right] \subseteq r_{A}^{p}\left(r_{A} r_{x}^{k} \dot{+} \cdots \dot{+} r_{A} r_{x} \dot{+} r_{A}\right) \subseteq r_{A}^{p+1} r_{x}^{k} \dot{+} \cdots \dot{+} r_{A}^{p+1} r_{x} \dot{+}\left(r_{A}\right)^{p+1}
$$

Thanks to the preceding lemma whe have for all integer $k, l, p$ and $q$ in $\mathbb{N}$ :

$$
r_{A}^{p} r_{x}^{k} \circ r_{A}^{q} r_{x}^{l} \subseteq r_{A}^{p+q} r_{x}^{k+l} \dot{+} \cdots \dot{+} r_{A}^{p+q} r_{x}^{l}
$$

Lemma 2.13. Let $L$ be a Leibniz algebra and $(l, r, V)$ a representation of $L$. Let $A$ be a subspace of the vector space $L$ and $x$ in the normalizer $n_{L}(A)$ of $A$ and for a non negative integer $k$ let $E_{k}$ be the subspace $E_{k}=r_{A} r_{x}^{k} \dot{+} \cdots \dot{+} r_{A}$. Then we have for all integer $p$ in $\mathbb{N}^{*}$ :

$$
E_{k}^{p} \subseteq r_{A}^{p} r_{x}^{p k} \dot{+} \cdots \dot{+} r_{A}^{p} r_{x}^{2 k} \dot{+} \cdots \dot{+} r_{A}^{p} r_{x} \dot{+} r_{A}^{p} \text {. }
$$

Proof. Let us compute $E_{k}^{p}$ for $p=2,3$; we have $\left[r_{x}, r_{a}\right]=r_{[a, x]}$, so

$$
\begin{aligned}
E_{k}^{2} & =\left(r_{A} r_{x}^{k} \dot{+} \cdots \dot{+} r_{A}\right)^{2}=\left(r_{A} r_{x}^{k} \dot{+} \cdots \dot{+} r_{A}\right)\left(r_{A} r_{x}^{k} \dot{+} \cdots \dot{+} r_{A}\right) \\
& \subseteq\left(r_{A} r_{x}^{k}\right)\left(r_{A} r_{x}^{k}\right) \dot{+} \cdots \dot{+} r_{A}\left(r_{A} r_{x}\right) \dot{+}\left(r_{A} r_{x}\right) r_{A} \dot{+} r_{A} r_{A} \subseteq r_{A}^{2} r_{x}^{2 k} \dot{+} \cdots \dot{+} r_{A}^{2} r_{x}^{k} \dot{+} \cdots \dot{+} r_{A}^{2} r_{x} \dot{+} r_{A}^{2}, \\
E_{k}^{3} & =\left(r_{A} r_{x}^{k} \dot{+} \cdots \dot{+} r_{A}\right)^{3}=\left(r_{A} r_{x}^{k} \dot{+} \cdots \dot{+} r_{A}\right)^{2}\left(r_{A} r_{x}^{k} \dot{+} \cdots \dot{+} r_{A}\right) \\
& \subseteq\left(r_{A}^{2} r_{x}^{2 k} \dot{+} \cdots \dot{+} r_{A}^{2} r_{x}^{k} \dot{+} \cdots \dot{+} r_{A}^{2}\right)\left(r_{A} r_{x}^{k} \dot{+} \cdots \dot{+} r_{A}\right) \subseteq\left(r_{A}^{2} r_{x}^{2 k}\right)\left(r_{A} r_{x}^{k}\right) \dot{+} \cdots \dot{+} r_{A}^{2}\left(r_{A} r_{x}\right) \dot{+}\left(r_{A}^{2} r_{x}\right) r_{A} \dot{+} r_{A}^{2} r_{A} \\
& \subseteq r_{A}^{3} r_{x}^{3 k} \dot{+} \cdots \dot{+} r_{A}^{3} r_{x}^{2 k} \dot{+} \cdots \dot{+} r_{A}^{3} r_{x} \dot{+} r_{A}^{3}
\end{aligned}
$$

and set by hypothesis that we have $E_{k}^{p-1} \subseteq r_{A}^{p-1} r_{x}^{(p-1) k} \dot{+} \cdots \dot{+} r_{A}^{p-1} r_{x} \dot{+} r_{A}^{p-1}$. 
The proof is then done.

So we will get $E_{k}^{p}=\left(r_{A} r_{x}^{k} \dot{+} \cdots \dot{+} r_{A}\right)^{p}=\left(r_{A} r_{x}^{k} \dot{+} \cdots \dot{+} r_{A}\right)^{p-1}\left(r_{A} r_{x}^{k} \dot{+} \cdots \dot{+} r_{A}\right)$

$$
\begin{aligned}
& \subseteq\left(r_{A}^{p-1} r_{x}^{(p-1) k} \dot{+} \cdots \dot{+} r_{A}^{p-1} r_{x} \dot{+} r_{A}^{p-1}\right)\left(r_{A} r_{x}^{k} \dot{+} \cdots \dot{+} r_{A}\right) \\
& \subseteq\left(r_{A}^{p-1} r_{x}^{(p-1) k}\right)\left(r_{A} r_{x}^{k}\right) \dot{+} \cdots \dot{+} r_{A}^{p-1}\left(r_{A} r_{x}\right) \dot{+}\left(r_{A}^{p-1} r_{x}\right) r_{A} \dot{+} r_{A}^{p-1} r_{A} \\
& \subseteq r_{A}^{p} r_{x}^{p k} \dot{+} \cdots \dot{+} r_{A}^{p} r_{x}^{2 k} \dot{+} \cdots \dot{+} r_{A}^{p} r_{x} \dot{+} r_{A}^{p} .
\end{aligned}
$$

Lemma 2.14. Let $L$ be a Leibniz algebra and $(l, r, V)$ a representation of $L$. Let $A$ be a subspace of the vector space $L$ and $x$ in the normalizer $n_{L}(A)$ of $A$. Let $m$ be a non negative integer. Then for all $(\lambda, a) \in F \times A$,

$$
f_{m}=\left(r_{a+\lambda x}\right)^{m}-\sum_{k=0}^{m}\left(\begin{array}{c}
m \\
k
\end{array}\right) \lambda^{k} r_{a}^{m-k} r_{x}^{k} \in r_{A} r_{x}^{m} \dot{+} \cdots \dot{+} r_{A} .
$$

Proof. By induction:

$$
f_{1}=\left(r_{a+\lambda x}\right)^{1}-\sum_{k=0}^{1}\left(\begin{array}{l}
1 \\
k
\end{array}\right) \lambda^{k} r_{a}^{1-k} r_{x}^{k}=r_{a+\lambda x}-\left(r_{a}+\lambda r_{x}\right)=0 \in r_{A} r_{x} \dot{+} r_{A} .
$$

And if by hypoyhesis we have: $f_{m}=\left(r_{a+\lambda x}\right)^{m}-\sum_{k=0}^{m}\left(\begin{array}{c}m \\ k\end{array}\right) \lambda^{k} r_{a}^{m-k} r_{x}^{k} \in r_{A} r_{x}^{m} \dot{+} \cdots \dot{+} r_{A}$, we can write:

$$
\begin{aligned}
& f_{m+1}=\left(r_{a}+\lambda r_{x}\right)^{m+1}-\sum_{k=0}^{m+1}\left(\begin{array}{c}
m+1 \\
k
\end{array}\right) \lambda^{k} r_{a}^{m-k+1} r_{x}^{k}=\left(r_{a}+\lambda r_{x}\right)^{m}\left(r_{a}+\lambda r_{x}\right)-\sum_{k=0}^{m+1}\left(\begin{array}{c}
m+1 \\
k
\end{array}\right) \lambda^{k} r_{a}^{m-k+1} r_{x}^{k} \\
& =\left(\sum_{k=0}^{m}\left(\begin{array}{c}
m \\
k
\end{array}\right) \lambda^{k} r_{a}^{m-k} r_{x}^{k}+f_{m}\right)\left(r_{a}+\lambda r_{x}\right)-\sum_{k=0}^{m+1}\left(\begin{array}{c}
m+1 \\
k
\end{array}\right) \lambda^{k} r_{a}^{m-k+1} r_{x}^{k} \\
& =\sum_{k=0}^{m}\left(\begin{array}{c}
m \\
k
\end{array}\right) \lambda^{k} r_{a}^{m-k} r_{x}^{k} r_{a}+f_{m} r_{a}+\sum_{k=0}^{m}\left(\begin{array}{c}
m \\
k
\end{array}\right) \lambda^{k+1} r_{a}^{m-k} r_{x}^{k+1}+\lambda f_{m} r_{x}-\sum_{k=0}^{m+1}\left(\begin{array}{c}
m+1 \\
k
\end{array}\right) \lambda^{k} r_{a}^{m-k+1} r_{x}^{k} .
\end{aligned}
$$

Then we have

$f_{m+1}=\sum_{k=0}^{m}\left(\begin{array}{c}m \\ k\end{array}\right) \lambda^{k} r_{a}^{m-k}\left(r_{x}^{k} r_{a}\right)+f_{m} r_{a}+\sum_{k=0}^{m}\left(\begin{array}{c}m \\ k\end{array}\right) \lambda^{k+1} r_{a}^{m-k} r_{x}^{k+1}+\lambda f_{m} r_{x}-\sum_{k=0}^{m+1}\left(\begin{array}{c}m+1 \\ k\end{array}\right) \lambda^{k} r_{a}^{m-k+1} r_{x}^{k}$.

Since $r_{x}^{k} r_{a}=r_{a} r_{x}^{k}+\beta_{k}$ we have

$$
\begin{gathered}
f_{m+1}=\sum_{k=0}^{m}\left(\begin{array}{c}
m \\
k
\end{array}\right) \lambda^{k} r_{a}^{m-k}\left(r_{a} r_{x}^{k}+\beta_{k}\right)+f_{m} r_{a}+\sum_{k=0}^{m}\left(\begin{array}{c}
m \\
k
\end{array}\right) \lambda^{k+1} r_{a}^{m-k} r_{x}^{k+1}+\lambda f_{m} r_{x}-\sum_{k=0}^{m+1}\left(\begin{array}{c}
m+1 \\
k
\end{array}\right) \lambda^{k} r_{a}^{m-k+1} r_{x}^{k} \\
=\sum_{k=0}^{m}\left(\begin{array}{c}
m \\
k
\end{array}\right) \lambda^{k} r_{a}^{m-k+1} r_{x}^{k}+\sum_{k=0}^{m}\left(\begin{array}{c}
m \\
k
\end{array}\right) \lambda^{k} r_{a}^{m-k} \beta_{k}+f_{m} r_{a} \\
\left.+\sum_{k=0}^{m}\left(\begin{array}{c}
m \\
k
\end{array}\right) \lambda^{k+1} r_{a}^{m-k} r_{x}^{k+1}+\lambda f_{m} r_{x}\right)-\sum_{k=0}^{m+1}\left(\begin{array}{c}
m+1 \\
k
\end{array}\right) \lambda^{k} r_{a}^{m-k+1} r_{x}^{k} \\
=r_{a}^{m+1}+\sum_{k=1}^{m}\left(\begin{array}{c}
m \\
k
\end{array}\right) \lambda^{k} r_{a}^{m-k+1} r_{x}^{k}+\sum_{k=0}^{m}\left(\begin{array}{c}
m \\
k
\end{array}\right) \lambda^{k} r_{a}^{m-k} \beta_{k}+f_{m} r_{a} \\
+\lambda^{m+1} r_{x}^{m+1}+\sum_{k=0}^{m-1}\left(\begin{array}{c}
m \\
k
\end{array}\right) \lambda^{k+1} r_{a}^{m-k} r_{x}^{k+1}+\lambda f_{m} r_{x}-\sum_{k=0}^{m+1}\left(\begin{array}{c}
m+1 \\
k
\end{array}\right) \lambda^{k} r_{a}^{m-k+1} r_{x}^{k} \\
=r_{a}^{m+1}+\sum_{j=1}^{m}\left(\begin{array}{c}
m \\
j
\end{array}\right) \lambda^{k} r_{a}^{m-j+1} r_{x}^{j}+\sum_{k=0}^{m}\left(\begin{array}{c}
m \\
k
\end{array}\right) \lambda^{k} r_{a}^{m-k} \beta_{k}+f_{m} r_{a} \\
+\lambda^{m+1} r_{x}^{m+1}+\sum_{j=1}^{m}\left(\begin{array}{c}
m \\
j-1
\end{array}\right) \lambda^{j} r_{a}^{m-j+1} r_{x}^{j}+\lambda f_{m} r_{x}-\sum_{k=0}^{m+1}\left(\begin{array}{c}
m+1 \\
k
\end{array}\right) \lambda^{k} r_{a}^{m-k+1} r_{x}^{k} \\
=r_{a}^{m+1}+\sum_{j=1}^{m}\left(\begin{array}{c}
m \\
j
\end{array}\right) \lambda^{k} r_{a}^{m-j+1} r_{x}^{j}+\sum_{j=1}^{m}\left(\begin{array}{c}
m \\
j-1
\end{array}\right) \lambda^{j} r_{a}^{m-j+1} r_{x}^{j}+\lambda^{m+1} r_{x}^{m+1} \\
-\left(\begin{array}{c}
\left.r_{a}^{m+1}+\sum_{k=1}^{m}\left(\begin{array}{c}
m+1 \\
k
\end{array}\right) \lambda^{k} r_{a}^{m-k+1} r_{x}^{k}+\lambda^{m+1} r_{x}^{m+1}\right)+\sum_{k=0}^{m}\left(\begin{array}{c}
m \\
k
\end{array}\right) \lambda^{k} r_{a}^{m-k} \beta_{k}+f_{m} r_{a}+\lambda f_{m} r_{x}
\end{array}\right.
\end{gathered}
$$


Finally we have

$$
\begin{aligned}
& f_{m+1}=\sum_{k=0}^{m}\left(\begin{array}{c}
m \\
k
\end{array}\right) \lambda^{k} r_{a}^{m-k} \beta_{k}+f_{m} r_{a}+\lambda f_{m} r_{x} \\
& \quad \in \sum_{k=0}^{m}\left(\begin{array}{c}
m \\
k
\end{array}\right) \lambda^{k}\left(r_{A}\right)^{m-k}\left(r_{A} r_{x}^{m} \dot{+} \cdots \dot{+} r_{A}\right) \dot{+}\left(r_{A} r_{x}^{m} \dot{+} \cdots \dot{+} r_{A}\right) r_{A} \dot{+} \lambda\left(r_{A} r_{x}^{m} \dot{+} \cdots \dot{+} r_{A}\right) r_{x} \\
& \quad \in r_{A} r_{x}^{m+1} \dot{+} \cdots \dot{+} \cdots \dot{+} r_{A} r_{x} \dot{+} r_{A} .
\end{aligned}
$$

Definition 2.15. Call $x \in \operatorname{End}(V)$ semisimple if the roots of its minimum polynomial over $F$ are all distinct, or equivalently, if $x$ is diagonalizable.

Remark 2.16. $\quad$ 1. Two commuting semisimple endomorphisms are simultaneously diagonalizable, so their sum and difference are both semisimple.

2. If $x$ is semisimple and $x$ leaves a subspace $W$ invariant, then the restriction of $x$ to $W$ denoted by $x_{\mid W}$ is semisimple.

Definition 2.17. Call $x \in L$ ad-semisimple (respectively Ad-semisimple) if the endomorphisms ad is $_{x}$ semisimple (respectively $A d_{x}$ is semisimple).

Call $x \in L$ ad-nilpotent (respectively Ad-nilpotent) if the endomorphisms ad $d_{x}$ is nilpotent (respectively $A d_{x}$ is nilpotent).

Lemma 2.18. Let $V=V_{1} \oplus V_{2}$ be a direct sum of two vector spaces $V_{1}, V_{2}$, an non negative integer $p$ and $\sigma$ an endomorphism of $V$ shuch that $\sigma^{p}(V) \subseteq V_{1}$, then the trace of $\sigma$ denoted by $\operatorname{tr}(\sigma)=\operatorname{tr}\left(\sigma_{\mid V_{1}}\right)$, where $\sigma_{\mid V_{1}}$ is the restriction of $\sigma$ to $V_{1}$.

Proof. Since we have an algebraically closed field, we can find a basis $\left\{v_{1}, \cdots, v_{m}, \cdots, v_{n}\right\}$ of $V$ whith $\left\{v_{1}, \cdots, v_{m},\right\}$ is a basis of $V_{1}$ and scalars $\lambda_{1}, \cdots, \lambda_{n}$ shuch that the matrix of $\sigma$ in this basis is

$$
N_{0 k}=\left(\begin{array}{ccccc}
\lambda_{1} & a_{1,2} & a_{1,3} & \ldots & a_{1, n} \\
0 & \lambda_{2} & a_{2,3} & \ldots & a_{2, n} \\
\vdots & 0 & \ddots & \ddots & \vdots \\
\vdots & \vdots & \ddots & \lambda_{n-1} & a_{n-1, n} \\
0 & 0 & \cdots & 0 & \lambda_{n}
\end{array}\right)
$$

For $m+1 \leq i \leq n$, we have a vector $0 \neq v_{i} \in V_{2}$ shuch that $\sigma\left(v_{i}\right)=\lambda_{i} v_{i}$.

Then $\sigma^{p}\left(v_{i}\right)=\lambda_{i}^{p} v_{i} \in V_{2} \cap V_{1}=\{0\}$. So $\lambda_{i}=0$ for $m+1 \leq i \leq n$, and

$$
\operatorname{tr}(\sigma)=\sum_{j=1}^{n} \lambda_{j}=\sum_{j=1}^{m} \lambda_{j}=\operatorname{tr}\left(\sigma_{\mid V_{1}}\right)
$$

\section{Radical and Nilradical}

The proof of following proposition can be found in [5].

Proposition 3.1. Let $\mathfrak{W}$ be a Lie subalgebra of $\operatorname{End}_{F}(V)$ where $V$ is an F-vector space. Then $\mathfrak{W}$ is solvable if and only if $\operatorname{tr}(x \circ y)=0$ for all $x \in \mathfrak{W}$ and $y \in[\mathfrak{W}, \mathfrak{W}]$.

Theorem 3.2. [1, Theorem 3.7] Let $L$ be a Leibniz algebra. Then $L$ is solvable if and only if for all $x$ in $L$ and all $y$ in $[L, L], \operatorname{tr}\left(a d_{x} \circ a d_{y}\right)=0$.

If $\imath$ is an ideal of $L$ and $L / \imath$ is solvable (respectively nilpotent), then $D^{(n)}(L / \imath)=0$ (respectively $(L / \imath)^{n}=0$ ) implies that $D^{(n)}(L) \subset \imath$ (respectively $L^{n} \subset \imath$ nilpotent). If $\imath$ itself is solvable with $D^{(m)}(\imath)=0$ (respectively nilpotent with $\imath^{m}=0$ ), then $D^{(m+n)}(L)=0$ (respectively $L^{m+n}=0$ ).

So we have proved: 
Proposition 3.3. If $\imath \subset L$ is an ideal, and both $\imath$ and $L / \imath$ are solvable (respectively nilpotent), so is $L$ solvable (respectively nilpotent).

If $\imath$ and $\jmath$ are solvable ideals, then $(\imath+\jmath) / \jmath \equiv \imath /(\imath \cap \jmath)$ is solvable, being the homomorphic image of a solvable algebra. So, by the previous propositio, we have the

Proposition 3.4. If $\imath$ and $\jmath$ are solvable ideals (respectively nilpotent ideals) in $L$ so $\imath+\jmath$ is solvable (respectively nilpotent). In particular, every Leibniz algebra $L$ has a largest solvable ideal which contains all other solvable ideals and a largest nilpotent ideal which contains all other nilpotent ideals.

The largest solvable one is denoted by $\operatorname{Rad}(L)$.

The largest nilpotent one is denoted by Nil $(L)$.

Remark 3.5. Note that $\operatorname{Ess}(L) \subseteq N i l(L) \subseteq \operatorname{Rad}(L)$.

\section{The ideal $\{\operatorname{Rad}(L), L\}$}

Let us denote the subspace $[\operatorname{Rad}(L), L] \dot{+}[L, \operatorname{Rad}(L)]$ by $\{\operatorname{Rad}(L), L\}$.

Lemma 4.1. Let $L$ be a Leibniz algebra and $(l, r, V)$ a representation of $L$. Let $A$ be a subspace of $L$ for which there exists an integer $n \in \mathbb{N}^{*}$ with $r_{A}^{n}=\{0\}$ and let $x$ be in $n_{L}(A)$ such that $r_{x}$ is nilpotent. Then there exists an integer $N \in \mathbb{N}^{*}$ with $\left(r_{A+F x}\right)^{N}=\{0\}$.

Proof. Let us notice that for any non negative integer $p$ we have

$\left(r_{a+\lambda x}\right)^{p}=\sum_{k=0}^{p}\left(\begin{array}{l}p \\ k\end{array}\right) \lambda^{k} r_{x}^{k}\left(r_{a}\right)^{p-k}+f_{p}$ where $f_{p} \in E_{p}=r_{A} r_{x}^{p} \dot{+} \cdots \dot{+} r_{A}$.

Let $m$ an integer with $\left(r_{x}\right)^{m}=0$. Then with $p=2 \sup (m, n)+1>m+n$ we have that $\left(r_{a+\lambda x}\right)^{p}=f_{p} \in E_{p}$. And so

$$
\left[\left(r_{a+\lambda x}\right)^{p}\right]^{n}=\left(f_{p}\right)^{n}=\left(r_{A} r_{x}^{p} \dot{+} \cdots \dot{+} r_{A}\right)^{n} \subseteq r_{A}^{n} r_{x}^{n p} \dot{+} \cdots \dot{+} r_{A}^{n} r_{x}^{2 p} \dot{+} \cdots \dot{+} r_{A}^{n} r_{x} \dot{+} r_{A}^{n} .
$$

Since $r_{A}^{n}=\{0\},\left(r_{a+\lambda x}\right)^{p n}=0$. So $r_{a+\lambda x}$ is nilpotent for all $a+\lambda x$ in $A \dot{+} F x$. By [7, Theorem 3.2., page 41] the associative algebra $r_{A \dot{+} F x}$ is nilpotent algebra. So there is some integer $N \in \mathbb{N}^{*}$ such that $\left(r_{A+F x}\right)^{N}=\{0\}$.

Proposition 4.2. For any representation $(l, r, V)$ of the Leibniz algebra $L$, the restriction of $r$ to the ideal $\{\operatorname{Rad}(L), L\}$ is nilpotent, i.e. there exists an integer $m \in \mathbb{N}^{*}$ with $\left(r_{\{\operatorname{Rad}(L), L\}}\right)^{m}=\{0\}$.

Proof. According to [3, Corollary 4.4] the representation of $V$ is nilpotent on the ideal $[L, L]$. Now let $T \subseteq$ $\{\operatorname{Rad}(L), L\}$ be a subspace containing $[\operatorname{Rad}(L), \operatorname{Rad}(L)]$, which is maximal with respect to the property that the representation of $V$ is nilpotent on $T$. Note that $T$ always is an ideal of $\operatorname{Rad}(L)$, hence in particular a subalgebra, because it contains $[\operatorname{Rad}(L), \operatorname{Rad}(L)]$.

Assume that $T \neq\{\operatorname{Rad}(L), L\}$. Then there exist at least an $x$ in $\operatorname{Rad}(L)$ and $y$ in $L$ with $[x, y] \notin T$ or $[y, x] \notin T$.

If $[x, y] \notin T$, the subspace $B=\operatorname{Rad}(L) \dot{+} F x$ is a subalgebra of $L, \operatorname{Rad}(L)$ is a solvable ideal of $B$ and $B / \operatorname{Rad}(L) \approx$ $F$ is abelian. Therefore $B$ is a solvable ideal by Proposition 3.3.

Again we use [3, Corollary 4.4] to see that the representation of $V$ is nilpotent on $[B, B]$ and hence that $r_{[x, y]}$ is nilpotent.

Since $T \subseteq \operatorname{Rad}(L)$ and $[x, y] \in[\operatorname{Rad}(L), y] \subseteq \operatorname{Rad}(L)$, we have $[[x, y], T] \subseteq[\operatorname{Rad}(L), T] \subseteq T$ and $[T,[x, y]] \subseteq[T, \operatorname{Rad}(L)] \subseteq T$.

Finally the preceding lemma show that the representation of $V$ is nilpotent on the subspace $T \oplus F[x, y]$. This contradicts the maximality of $T$.

If $[y, x] \notin T$, the subspace $B=\operatorname{Rad}(L) \dot{+} F x$ is a subalgebra of $L, \operatorname{Rad}(L)$ is a solvable ideal of $B$ and $B / \operatorname{Rad}(L) \approx$ $F$ is abelian. Therefore $B$ is a solvable ideal by Proposition 3.3.

Again we use [3, Corollary 4.4] to see that the representation of $V$ is nilpotent on $[B, B]$ and hence that $r_{[y, x]}$ is nilpotent.

Since $T \subseteq \operatorname{Rad}(L)$ and $[y, x] \in[y, \operatorname{Rad}(L)] \subseteq \operatorname{Rad}(L)$, we have $[[y, x], T] \subseteq[\operatorname{Rad}(L), T] \subseteq T$ and $[[y, x], T] \subseteq[\operatorname{Rad}(L), T] \subseteq T$.

Finally the preceding lemma show that the representation of $V$ is nilpotent on the subspace $T \oplus F[x, y]$. This contradicts the maximality of $T$. 
We conclude that $T$ must be equal to $\{\operatorname{Rad}(L), L\}$, so the representation of $V$ is nilpotent on $\{\operatorname{Rad}(L), L\}$.

Applying the precedent proposition to the adjoint representation $(A d, a d, L)$ of the Leibniz algebra $L$ and using Engel's Theorem [2], we get the:

Corollary 4.3. The ideal $\{\operatorname{Rad}(L), L\}$ is nilpotent. In particular, $x$ is ad-nilpotent for every $x$ in $\{\operatorname{Rad}(L), L\}$.

Corollary 4.4. Let $L$ be a Leibniz algebra and $D$ a derivation of $L$.

Then $D(\operatorname{Rad}(L)) \subseteq N i l(L)$. In particular $N i l(L)$ is a characteristical ideal.

Proof. For a derivation $D$ of $L$, define the Leibniz algebra $\tilde{L}=L \times\left.\right|_{D} F$ with the bracket $[(x, t),(y, l)]=(l D(x)-t D(y)+[x, y], 0)$. Then,

$(D(\operatorname{Rad}(L)), 0)=[(\operatorname{Rad}(L), 0)(0,1)] \subseteq(L, 0) \cap[\operatorname{Rad}(\tilde{L}), \tilde{L}] \subseteq \tilde{L} \cap \operatorname{Nil}(\tilde{L}) \subseteq \operatorname{Nil}(\tilde{L})=(\operatorname{nil}(L), 0)$. So $D(\operatorname{Rad}(L)) \subseteq N i l(L)$.

\section{Main theorem}

We deal in this section with Leibniz algebras which sastify equation

$\forall x, y \in L, \operatorname{tr}\left(a d_{x} \circ a d_{y}\right)_{\mid E s s(L)}=0$

Call such Leibniz algebras: Killing Leibniz Algebras.

A bilinear form $(-,-): L \times L \longrightarrow F$ is called invariant if $([x, y], z)+(y,[x, z])=0$ for all $x, y, z$ in $L$.

Notice that if $(-,-)$ is an invariant form, and $\imath$ is an ideal, then its orthogonal $\imath^{\perp}$ is again an ideal.

One way of producing invariant forms is from representations: if $(l, r, V)$ is a representation of $L$, then $(x, y)_{r}=\operatorname{tr}\left(r_{x} \circ r_{y}\right)$ is invariant. Indeed,

$([x, y], z)_{r}+(y,[x, z])_{r}=\operatorname{tr}\left(\left(r_{y} \circ r_{x}-r_{x} \circ r_{y}\right) \circ r_{z}+r_{y} \circ\left(r_{z} \circ r_{x}-r_{x} \circ r_{z}\right)\right)$

$$
=\operatorname{tr}\left(\left(r_{y} \circ r_{z}\right) \circ r_{x}-r_{x} \circ\left(r_{y} \circ r_{z}\right)\right)=0 .
$$

In particular, if we take $l=A d, r=a d, V=L$ the corresponding bilinear form is called the Killing form and will be denoted by $\mathfrak{K}=(-,-)_{\mathfrak{K}}$.

Remark 5.1. for all $x$ in $\operatorname{Ess}(L), y, z$ in $L$ we have: $\left(a d_{x} \circ a d_{y}\right)(z)=\left(a d_{x}\right)([z, y])=[[z, y], x]=0$.

Then $a d_{x} \circ a d_{y} \equiv 0$ and $(x, y)_{\mathfrak{K}}=\operatorname{tr}\left(a d_{x} \circ a d_{y}\right)=0$, so $\operatorname{Ess}(L) \subseteq \operatorname{ker}(\mathfrak{K})$.

Theorem 5.2. Let L be a leibniz algebra of a class Killing Leibniz Algebras and $\operatorname{ker}(\mathfrak{K})$ the kernel of its Killing form.

$$
\operatorname{ker}(\mathfrak{K})=\operatorname{Ess}(L) \Leftrightarrow L \text { is semisimple. }
$$

Proof. $\Leftarrow$ Suppose that $L$ is semisimple. Let us show that the kernel of the Killing form is $\operatorname{Ess}(g)$.

So let $\mathfrak{W}=L^{\perp}=\left\{x \in L, \operatorname{tr}\left(a d_{x} \circ a d_{y}\right)=0\right.$ for all $\left.y \in L\right\}$. If $x \in \mathfrak{W}, y, z \in L$ then

$\operatorname{tr}\left(a d_{[x, z]} \circ a d_{y}\right)=\operatorname{tr}\left(a d_{x} \circ a d_{z} \circ a d_{y}-a d_{z} \circ a d_{x} \circ a d_{y}\right)=\operatorname{tr}\left(a d_{x} \circ\left(a d_{z} \circ a d_{y}-a d_{y} \circ a d_{z}\right)\right)$

$$
=\operatorname{tr}\left(a d_{x} \circ a d_{[z, y]}\right)=0,
$$

And so on, we have also $\operatorname{tr}\left(a d_{[z, x]} \circ a d_{y}\right)=0$.

So $\mathfrak{W}$ is an ideal and clearly $\operatorname{Ess}(L) \subseteq \mathfrak{W}$.

$a d_{\mathfrak{W}}$ is a solvable a Lie subalgebra of $\operatorname{End}(V)$ by Cartan's criterion. Thanks to Proposition 3.1, $\mathfrak{W}$ is solvable and hence $\mathfrak{W}=\operatorname{Rad}(L)=\operatorname{Ess}(L)$.

$\Rightarrow$ suppose $L$ is not semisimple and so has a solvable ideal such that $a \supsetneq E s s(L) \supseteq a^{2}$ by Remark 2.8. Let us show that $(x, y)_{K}=0$ for all $x$ in $a, y$ in $L$ and then $a \subset \operatorname{ker}(\mathfrak{K})$.

Let $\sigma=a d_{x} \circ a d_{y}$.

By assumption $\operatorname{tr}\left(\sigma_{\mid E s s(L)}\right)=0$.

And since $\sigma$ maps $L$ to $a, a$ to $a^{2}$ and $a^{2} \subseteq \operatorname{Ess}(L)$, we have that

$\sigma^{2}(L) \subseteq \sigma(a) \subseteq a^{2} \subseteq \operatorname{Ess}(L)$.

Write $L=\operatorname{Ess}(L) \oplus L_{2}$. Then we have by Lemma 2.18, that $\operatorname{tr}(\sigma)=\operatorname{tr}\left(\sigma_{\mid E s s(L)}\right)=0$. Hence if $L$ is not semisimple then the kernel of its Killing form satisfies $\operatorname{Ess}(L) \subsetneq \operatorname{ker}(\mathfrak{K})$. 
Remark 5.3. - I. Demir et al. give another proof of $\Rightarrow$. (see [4, Theorem 5.8]).

- In Lie algebras case, Theorem 5.2 is the well known Cartan's criterion for semisimplicity.

\section{Conclusion}

Let us cite an example of Leibniz algebra which is solvable and the kernel of it's Killing form is $\operatorname{Ess}(L)$.

Example 6.1. $[6]$

Let $L=\mathbb{C} x+\mathbb{C} y$ be the two dimensional complex Leibniz algebra which generators satisfy $[x, y]=x$; $[x, x]=[y, y]=[y, x]=0$.

Let us find the kernel of the Killing form of the non lie leibniz algebra $L=F x \oplus F y$ defined in Example 6.1. Let $a=a_{11} x+a_{12} y$ and $b=a_{21} x+a_{22} y$ be two elements of algebra. The matrix of the endomorphism $a d_{a}$ is $\left(\begin{array}{cc}a_{12} & 0 \\ 0 & 0\end{array}\right)$ and the matrix of the endomorphism $a d_{b}$ is $\left(\begin{array}{cc}a_{22} & 0 \\ 0 & 0\end{array}\right)$.

Then the Killing form is defined by $(a, b)_{\mathfrak{K}}=a_{12} a_{22}$ for all $a, b$ in $L$.

Since $\operatorname{Ess}(L)=\{0\}$ for any Lie algebra; Lie algebras are Killing Leibniz algebras and the Theorem 5.2 is knowned for Lie algebras (cf. [5]).

"Left central Leibniz" are also Killing Leibniz algebras.

Example 6.1 is an algebra not in a class of Killing Leibniz algebras.

We claim that

Claim: The class of Killing Leibniz Algebras is a widest class wich satisfies Theorem 5.2.

In [6], the authors call an algebra that is both a left and right Leibniz algebra a symmetric Leibniz algebra. they call $\mathrm{L}$ a left central Leibniz algebra if it is a left Leibniz algebra that also satisfies $[[a, a], b]=0, a \in L, b \in L$. There is a hierarchy of algebras $\{$ leftLeibniz $\} \supsetneq\{$ leftcentralLeibniz $\} \supsetneq\{$ symmetricLeibniz $\} \supsetneq\{$ Lie $\}$.

We call a right central Leibniz algebra if it is a right Leibniz algebra that also satisfies $[b,[a, a]]=0, a \in L, b \in L$ and there is a hierarchy of algebras

$$
\{\text { rightLeibniz }\} \supsetneq\{\text { rightcentralLeibniz }\} \supsetneq\{\text { symmetricLeibniz }\} \supsetneq\{\text { Lie }\} \text {. }
$$

So we can complete the hierarchy of Leibniz algebras as

$$
\{\text { rightLeibniz }\} \supsetneq\{\text { rightKillingLeibniz }\} \supsetneq\{\text { rightcentralLeibniz }\} \supsetneq\{\text { symmetricLeibniz }\} \supsetneq\{\text { Lie }\} .
$$

and

$$
\{\text { leftLeibniz }\} \supsetneq\{\text { leftKillingLeibniz }\} \supsetneq\{\text { leftcentralLeibniz }\} \supsetneq\{\text { symmetricLeibniz }\} \supsetneq\{\text { Lie }\} .
$$

\section{Questions:}

- Can we prove the Weyl's theorem on complete reducibility for Killing Leibniz Algebras?

- In [6], the authors show that "left central Leibniz algebras" satisfy a version of the Malcev theorem. Do the Killing Leibniz Algebras also satisfy this theorem?

\section{References}

[1] S., Ayupov, Sh.A. and Omirov, B.A.,," Cartan subalgebras, weight spaces and criterion of solvability of finite dimensional Leibniz algebras.", Rev. Mat. Complut., Vol.19, No.1, (2006), pp.183-195.

[2] D. W. Barnes, "On Engel's theorem for Leibniz algebras", Comm. Algebra, Vol.40, No.1, (2012), 1388-1389, arXiv:1012.0608.

[3] C. J. A. Béré, N . B. Pilabré and A. Kobmbaye "Lie's theorems on soluble Leibniz algebras.", British journal of Mathematics \&6 Computer Science, Vol.4, No.18, (2014), pp.2570-2581.

[4] I. Demir, K. C. Misra and E. Stitzinger "On some structures of Leibniz Algebras, ", arXiv:1307.7672v1 . 
[5] J. E. Hymphreys, Introduction to Lie Algebras and Representation Theory, Graduate Texts in Mathematics) Springer, (1972). http://books.google.bf/books?id=SD4DvUFa6QC

[6] G. Masons and G. Yamskulna, "Leibniz Algebras and Lie Algebras", Published online October 23, 2013 http://dx. doi.org/10. 3842/SIGMA.2013.063.,

[7] R. Schafer, Non associative algebras, https://www.gutenberg.org/files/25156/25156-pdf .pdf 